and distributed by the 'Tiers Monde' section of the Association des Documentalistes et Bibliothécaires Spécialisés (ADBS). Information is presented in the form of cards which may be cut up for filing. Basic annual subsctiption rate is $\mathrm{F}_{32}$; further information may be obtained from CARDAN, c/o EPHE, 20, tue de Baume, 75-Paris 8 .

\title{
African Bibliographic Center, Washington D.C.
}

THE monthly journal of the African Bibliographic Center (A Current Bibliography on African Affairs, published by Greenwood Periodicals, Inc., 2 I I Eașt 43rd Street, New York, N.Y. 10017) contains two new subject headings: 'African Heritage Studies' which lists and describes selected materials on those of African descent abroad, and 'Instruction Materials ' which is a survey of the growing body of literature and other materials designed for school and university instruction. The journal is being complemented by special bibliographical guides on both themes in the Special Bibliographic Series and Current Reading List Series. Additional information may be obtained from the Center, P.O. Box 13096, Washington D.C. 20009, U.S.A.

\section{'Bibliographie du Tchad (Sciences humaines)'}

Bibliographie $d u$ Tchad (sciences humaines) est le fruit d'un travail collectif poursuivi à FortLamy, à l'Institut National Tchadien pour les Sciences humaines, et à Paris, au Laboratoire d'ethnologie et d'Archéologie tchadiennes et camerounaises, dans le cadre de la R.C.P. II7 du CNRS. Arrêtée au $3 \mathrm{I}$ decembre 1967 , elle est consacrée aux textes relevant des sciences de l'homme et à ceux qui, traitant des sciences de la nature, la géologie, par exemple, presentent un intérêt particulier pour les premières, paléontologie et archéologie, entre autres. La bibliographie à été limitée aux publications imprimées ou ronéotées, aux disques et aux films. La nomenclature des cartes fera l'objet d'une publication spéciale établie sous la direction de $M$. J. Cabot, Professeur à l'Université de Vincennes, responsable de la Section de Géographie nouvellement créée à l'Institut National Tchadien pour les Sciences humaines. De même, l'inventaire des documents d'archives interessant le Tchad rassemblés tant au Depôt Officiel d'Archives de Fort-Lamy qu'à la section d'Outre-mer des Archives Nationales, mis en route par M. A. Fierro, continué par M. M. Vaisbrot, archivistespaléographes, est en cours d'établissement.

Prix de vente de la Bibliographie du Tchad: FIs. Adresser toute la correspondance à: Fort-Lamy-I.N.T.S.H., B.P. 503 ou Paris $\left(9^{\circ}\right)$ - I.N.T.S.H., 8, rue Henner. 